\title{
Educação para a Saúde Bucal: trabalho em equipe e aspectos psicossociais
}

\author{
Dental Health Education: Team Work and Psychosocial Factors
}

Iris Sawazaki *

Luiza Nakama $*$ *

SAWAZAKI, I, NAKAMA, L. Educação para a Saúde Bucal: Trabalho em Equipe e Aspectos

Psicossociais. Semina, Londrina, v. 18, ed. especial, p. 15-24, fcv. 1997.

RESUMO: A educação é o ponto essencial de qualquer programa de saúde. Seus resultados são significativos quando conseguem promover mudanças positivas no comportamenio das pessoas. O presente trabalho visa realizar um estudo sobre os aspectos psicossociais envolvidos na mudança comportamental das pessoas em relação à saúde bucal, relacionando-os com o trabalho educativo-preventivo de cárie dentária realizado pela equipe odontológica, bem como a compatibilidade deste trabalho com o programa da Secretaria de Saúde do municipio de Londrina.

A amostra constituiu-se de 433 pais de crianças de 0 a 14 anos atendidas na rede de Unidades Básicas de Saúde (UBS) do município de Londrina, 107 profissionais do serviço odontológico da rede, e seu respectivo coordenador. Todas as respostas foram obtidas mediante a aplicação de questionários.

Os resultados mostram que o trabalho educativo-preventivo de cárie dentária realizado pelos profissionais está de acordo com o proposto pela Secretaria de Saúde do municipio, e tem produzido reflexos positivos no saber e comportamento das pessoas com relação à saúde bucal.

Sugere-se o incentivo à formação e inserção de maior número de Técnicos de Higiene Dental e Auxiliares de Consultório Dentário em funções específicas de educação para a saúde, para maior cobertura da população, a baixo custo para o municipio.

PALAVRAS-CHAVE: Cárie Dentária/Prevenção e Controle; Educação em Saúde Bucal; Equipe de Assistência ao Paciente.

* Autora: Aluna do $5^{\circ}$ ano de Gradarąão em Odontologia da Universidade Estadual de Londrina.

\#: Orientadora: Mestre en Saúde Coleriva, Prof Assistente de Odontopediatria da Universidade Estadual de Londrina/Centro de Ciências da Saúde-

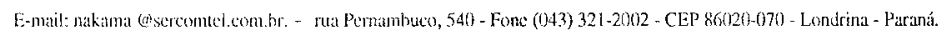


ABSTRACT: Education is the essential point of any health program. Results are significant when they promote positive changes in human behaviour. The present study is about psychosocial factors involved in the change of human behavior related to oral health, connecting this change with dental caries educational -preventive work realized by the dental team work, as well as the compatibility of this work with Londrina s Health Secretary program.

The sample consisted of 433 parents of children aged 0 to 14 years old attended by the net of Health Basic Units of Londrina, 107 professionals of the dental services provided in this network and their respective co-ordinator. All the answers were obtained by the application of questionnaires.

The results show that the educational-preventive work of dental caries realized by the professionals agree with that proposed by the Health Secretary, and have produced positive reflections on the knowledge and behavior of the people about oral health.

The findings suggest the incentive to the formation and the introduction of a higher number of dental therapists and dental auxiliaries for the higher assistance coverage of the population with low costs to the city.

KEY WORDS: Dental Caries; Prevention \& control; Dental Health Education; Patient Care Team.

\section{INTRODUÇÃO}

Em 1981, Londrina estava com um índice CPOD médio de 6.9 aos 12 anos de idadc. No ano de 1995, cste índice caiu para 3.1 , praticamente alcançando o preconizado pela Organização Mundial de Saúde (CPOD 3.0), para o ano 2000.

Essa queda pode ser atribuída à fluoretação das águas do sistema de abastecimento iniciada $\mathrm{cm}$ alguns locais en 1972, sendo que, a cobertura total do município completou-se em 1990. Além desse fato, cumpre ressaltar o papel de outros fatores como: a municipalização dos serviços de saúde, a priorização do atendimento odontológico de 0 a 14 anos nas Unidades Básicas de Saúde (UBS) a partir do ano de 1990, a implantação da Bebê-Clínica/UEL, com atendimento de (0) 5 anos desde 1986, c o uso de dentífricios fluoretados.
Fazendo uma projeção da capacidade de atendimento das UBSs e da Bebê-Clínica/UEL, as duas maiores instituições públicas de atendimento educativopreventivo programado do município, verifica -se que, diante de uma população de 130.693 crianças de 0 a 14 anos (IBGE, 1995), as duas instituições juntas têm capacidade para atender $40 \%$ desta população. Questiona-se então, qual seria a proposta custo-bencfício mais viável para se tentar atingir os $60 \%$ das crianças que não têm acesso ao serviço odontológico gratuito e, de uma forma otimista, atingir também uma parcela da população adulta.

A educação, já existente nos programas de prevenção destas duas instituições, é uma das soluções possíveis. FEJERSKOV (1995), num relato a respeito do declínio de cáries na Dinamarca, evidencia a educação como a 
causa principal desta queda. Nesta mesma linha, outros artigos da Islândia (1) mostram um declínio dramático de cáries, num curto período de tempo, onde o fornecimento de flúor não teria aparentemente mudado, mas crianças e adultos haviam sido bombardeados com informações e haviam se engajado no controle da doença.

O processo de educação envolve, sobretudo, a mudança comportamental. É importante detectar os pontos favoráveis e as dificuldades neste processo, sem perder de vista que este envolve responsabilidades da equipe odontológica, comunidade e entidades governamentais(6).

O presente trabalho visa, portanto, realizar um estudo sobre os seguintes aspectos:

a) Fatores psicossociais que envolvem a mudança comportamental em relação à saúde bucal dos usuários das UBSs do município de Londrina;

b) Facilidades e dificuldades no processo de ensinoaprendizagem entre o usuário e a equipe odontológica, ben como os reflexos deste trabalho no comportamento e saber das pessoas;

c) A relação entre o programa da Secretaria de Saúde do município e o trabalho educativo-preventivo realizado pela equipe odontológica das UBSs, procurando apontar novos caminhos ná busca da manutenção da saúde bucal no município de Londrina.

\section{METODOLOGIA}

O universo de pesquisa abrange as Unidades Básicas de Saúde da Prefeitura, á maior instituição pública vinculada à atenção à saúde bucal de 0 a 14 anos de idade no município de Londrina. A população cstudada compreende: usuários, profissionais e coordenador do serviço odontológico das Unidades Básicas de Saúde (UBS) do Município de Londrina.
A amostra de usuários foi realizada considerando-se as 24 UBSs da zona urbana que mantêm serviço de atenção odontológica.

Realizado o agrupamento, baseado $\mathrm{cm}$ características sócio-econômico-culturais da população da área de abrangência* , foi feito um sorteio aleatório de 11 UBSs onde foram aplicados 440 questionários, sendo respondidos 433 pelos pais das crianças em atendimento e 07 considerados perdidos, pois estavam incompletos ou rasurados. Quanto aos profissionais das UBSs, foram aplicados questionários a toda a equipe do serviço odontológico de todas as UBSs da região urbana, totalizando ao final 107 questionários e 1 questionário referente ao coordenador do Departamento de Unidades Básicas de Saúde (DUBS) da Secretaria de Saúde.

Todo o processo de seleção da amostra foi oticntado $\mathrm{c}$ acompanhado pelo Núcleo de Epidemiologia Clínica do Centro de Ciências de Saúde (CCS) da UEL .

Os dados foram coletados através de questionários com questões abertas e fechadas, de acordo com a proposta de metodologia quanti-qualitativa, tendo sido previamente testados e validados.

As questões formuladas aos usuários diziam respeito ao seu conhecimento geral sobre prevenção de cárie dentária, onde recebeu estas informações, tipo de informações recebidas, modificações de hábitos na família e na criança, importância que a pessoa atribui à prevenção de cáries, bem como sua caracterização sócioeconômica.

As questões dirigidas à equipe odontológica das UBSs e seu coordenador foram propositalmente semelhantes para que, na análise, pudessem ser comparadas as opiniõcs, e eram relativas a tipos de procedimentos educativo-preventivos realizados e assuntos abordados na orientação aos usuários, bem como a opinião sobre a eficácia de seu trabalho.

* A caracterização sócio-econômico-cultural foi realizada segundo o Relatótio Geral do Processo de Territorializaçāo do Município de Londritua, mar. 1995. 
Os dados coletados foram arquivados e consolidados no banco de dados do EPI INFO6, v.6.02.

\section{RESULTADOS E DISCUSSÃO}

Os dados colctados da amostra de usuários serão analisados de acordo com aspectos psicossociais (caracterização sócio-econômica, grupos de pressão e mudança comportamental, percepções e crenças, conhecimento e informação,) que interferem na mudança de comportamento das pessoas no que se refere à saúde bucal(7), usando a cárie dentária como assunto estratégico para avaliar estes aspectos, pois é a doença prevalente na população atcndida pelas UBSs, sendo as orientações dadas também aplicáveis à prevenção da doença periodontal.

Os profissionais serão analisados em relação ao proposto pelo programa de rotina educativo-preventiva da Secretaria de Saúde do Município(i6) e em relação a sua atuação nos conhecimentos e informações dos usuários.

\subsection{CARACTERIZAÇÃO SÓCIO-ECONÔMICA}

A caracterização sócio-econômica é de fundamental importancia quando se analisa mudança de comportamento, pois aspectos culturais, educacionais, econômicos e sociais são importantes para a delinitação do problema, suas possíveis soluções, barreiras à comunicação e outros aspectos ligados ao processo(10).

Os usuários são caracterizados sócio-economicamente pela predominância de pais com $1^{\circ}$ grau incompleto e renda familiar entre 3 a 4 salários mínimos, conforme tabelas 1 e 2, o que diferc dos usuários da BebêClínica/UEL, onde, segundo NAKAMA (1994), há predominância do nível de escolaridade entre $2^{\circ}$ e $3^{\circ}$ grau completo e renda familiar de 3 a 5 salários mínimos. Estes dados sugerem que, apesar da maioria da população usuária das UBSs ter menor nível de escolaridade e menor renda familiar em relação aos usuários da BebêClínica, a preocupação com a saúde bucal de suas crianças é a mesma nos dois grupos.

TABELA - Distribuição dos pais das crianças $\mathrm{cm}$ atendimento nas UBSs quanto ao grau de escolaridade.

\begin{tabular}{lcc}
\hline Escolaridade & Freq.absol. & Freq. Helat. \\
\hline Não estudou & 10 & $2,3 \%$ \\
\hline $1^{\circ}$ grau incompleto & 158 & $36,5 \%$ \\
\hline $1^{\circ}$ grau completo e & & $30,0 \%$ \\
\hline $2^{\circ}$ grau incompleto & 130 & $16,8 \%$ \\
\hline $2^{\circ}$ grau completo e & & \\
curso técnico & 73 &
\end{tabular}

$3^{\circ}$ grau incompleto e

\begin{tabular}{lcc}
$3^{\circ}$ grau completo & 37 & $8,5 \%$ \\
\hline Não responde & 16 & $3,7 \%$ \\
\hline Não sabe & 09 & $2,1 \%$ \\
\hline TOTAL & 433 & $100,0 \%$ \\
\hline
\end{tabular}

TABELA 2 - Distribuição das respostas dos pais das crianças atendidas na UBSs quanto à renda familiar

\begin{tabular}{llc}
\hline Renda familiag: & Freq.absol & Freq. relat. \\
\hline Até 2 SM & 82 & $18,9 \%$ \\
\hline 3 a 4 SM & 202 & $46,6 \%$ \\
\hline 5 SM a mais & 90 & $\frac{20,8 \%}{3,2 \%}$ \\
\hline Não responde & 14 & $\frac{10,4 \%}{100,0 \%}$ \\
\hline Não sabe & 45 & Fonte: Londrina, 1996 \\
\hline TOTAL & 433 &
\end{tabular}

\subsection{PERCEPÇÕES E CRENÇAS}

O modo pelo qual se vê as pessoas, situações e objetos na vida humana é chamado percepção. O 
comportamento em qualquer situação é determinado principalmente pelas crenças, atitudes e valores que possuímos $(10)$.

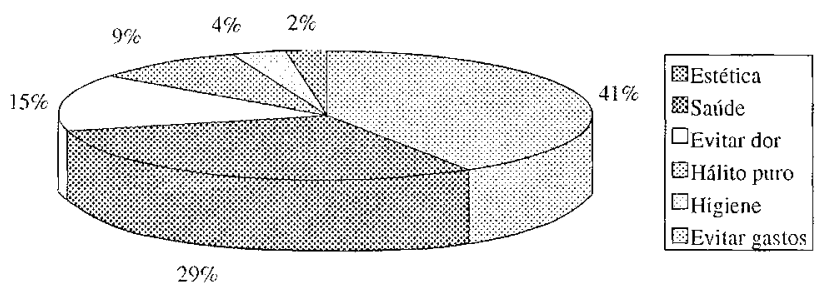

FIGURA 1 - Motivos que levam a população a procurar atendimento odontológico

Seguindo esta linha de raciocínio, analisa-se, a partir da figura 1 (anexo), o valor que as pessoas dão à saúde bucal, sendo que $99,5 \%$ a consideram importante e atribuem esta importância a fatores como: estética (41\%), saúde $(29 \%)$, evitar a dor (15\%), ter bom hálito $(9 \%)$ seguido por higiene (4\%) e evitar gastos $(2 \%)$. Estes dados revelam algumas das forças motivadoras que lcvam esta população a procurar orientações e ou atendimento educativo-preventivo de cárie dentária. 0 fato da estética ter sido tão citada pode ser um dos retlexos da mídia e das próprias exigências do mercado de trabalho, evidenciando que as necessidades humanas podem ser resultantes de fatores sociais e não somente biológicos(12). Estes dados sugerem que, apesar da estética ser o principal atrativo, a saúde bucal deve ser mais enfatizada pelos prolissionais da equipe odontologica, a fim de cstabelecer-se a real necessidade de prevenção dessa doença.

Na labcla 3, analisando a percepção das pessoas quanto à possibilidade de ter clentes sem cáries a vida toda, apenas $52,4 \%$ acham possivel, mostrando que as pessoas ainda possuem a crença de que a cárie é uma fatalidade. Este fato é negativo à medida que estimula o comportamento conformista, ou seja, "por que tanto esforço em prevenir se eu vou ter cáries mesmo?". PINTO (1992) relata que, na maior parte do países latinoamericanos, "a cárie dentária seguida pelas extrações e pela prótese, representam uma fatalidade inevitável ou uma contingência natural ligada à carência financeira", situação esta que pode encaixar-se na população em estudo, já que a sua grande maioria tem a renda familiar baixa( em torno de 3 a 4 salários mínimos).

TABELA 3-Distribuição das respostas dos usuários quanto a possibilidade de terem dentes sem cáries a vida toda

\section{Acha possível}

ter dentes sem

cáries a vida

\begin{tabular}{|c|c|c|}
\hline Loda & Freq. absol. & Freq. relat. \\
\hline $\operatorname{Sim}$ & 227 & $52,4 \%$ \\
\hline Não & 164 & $37,9 \%$ \\
\hline Não responde & 05 & $1,2 \%$ \\
\hline Não sabe & 37 & $8,5 \%$ \\
\hline TOTAL & 433 & $100 \%$ \\
\hline
\end{tabular}

Fonte: Zondrina, 1996

\subsection{CONHECIMENTOS EINFORMACÃO}

"Conhecimento é a palavra chave. É o poder de um individuo, de um grupo ou de uma comunidade."(6)

Na tabela 4 pode-se observar que $95,6 \%$ dos pais das crianças atendidas nas UBSs, já receberam algum tipo de orientação e ou atendimento educativo-preventivo, concordando com a grande proporção de profissionais da equipe odontológica das UBSs que afirman realizar algum tipo de atendimento educativo-preventivo (tabela 5).

Verifica-se nessa tabela, a importância do papel dos THDs e ACDs junto ao cirurgião-dentista no trabalho educativo-preventivo, visto que $100 \%$ dos THDs c $83,3 \%$ dos $\mathrm{ACD}$ realizam este atendimento. Isto é bastante 
promissor, principalmente quando se analisa iniciativas como a da Suécia,da Nova Zelândia c de outros países, onde houve quedas concretas no índice de cárie e doença periodontal com a atuação destes profissionais(4).

As tabelas 4 e 5 , mostram, portanto, concordâneia entrc a atuação dos profissionais da rede e as diretrizcs da Secretaria de Saúde, que priorizam uma linha de atuação educativo-preventiva.

TABELA 4 - Distribuição das respostas dos usuários que já receberam algum tipo de orientação e ou atendimento educativo-preventivo de cárie dentária

\begin{tabular}{lll}
$\begin{array}{c}\text { Já recebcu algum } \\
\text { tipo de } \\
\text { orientação } \mathrm{e} \text { ou } \\
\text { atendimento }\end{array}$ & Freq. absol. & Freq. relat. \\
\hline $\mathrm{Sim}$ & $\frac{414}{19}$ & $\frac{95,6 \%}{4,4 \%}$ \\
\hline Não & $\frac{19}{433}$ & $100,0 \%$ \\
\hline TOTAL & Fonte: Londrina. 1996
\end{tabular}

TABELA 5 - Distribuição do número de profissionais que compõem a cquipe odontológica que realiza algum tipo de trabalho educativo preventivo

\begin{tabular}{|c|c|c|c|c|c|c|}
\hline $\begin{array}{l}\text { Realiza algum } \\
\text { tipo de traballho } \\
\text { educativo- } \\
\text { preventivo }\end{array}$ & $\begin{array}{l}C D \\
\text { Freq. } \\
\text { absol. }\end{array}$ & $\begin{array}{l}\text { Freq. } \\
\text { relat. }\end{array}$ & $\begin{array}{l}\text { TH } \\
\text { Freq. } \\
\text { absol. }\end{array}$ & $\begin{array}{l}\text { D } \\
\text { Freq. } \\
\text { relat. }\end{array}$ & $\begin{array}{r}\mathrm{AC} \\
\text { Freq. } \\
\text { absol. }\end{array}$ & $\begin{array}{l}\text { D } \\
\text { Freq. } \\
\text { relat. }\end{array}$ \\
\hline $\operatorname{Sim}$ & 32 & $100 \%$ & 21 & $100 \%$ & 45 & $83.3 \%$ \\
\hline Não & 0 & 0 & 0 & 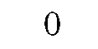 & 9 & $16.7 \%$ \\
\hline TOTAL & 32 & $100 \%$ & 21 & $100 \%$ & 54 & $100 \%$ \\
\hline
\end{tabular}

CD - Commsano-Demista

THD - Téchicos em Irigiene Denal

ACD -Akndeme de Comsutronio Demairio

Fontc: Londrina, 1996

Quando os pais são indagados a respeito do tipo de orientação c ou atcndimento educativo-preventivo que receberam para a saúde bucal de sua criança(tabela 7), $91,1 \%$ citam que receberam orientação de como escovar/limpar os dentes, seguidos por $61,4 \%$ que foram instruídos a como passar o fio dental, proporção de respostas cstas compatíveis com aquelas citadas pelos profissionais (tabela 6) que orientam quanto a instrução de higiene e escovação supervisionada, $87,8 \%$ e $72,4 \%$
TABELA 6 - Distribuição dos tipos de orientaçôes e ou atendimento dentário realizado pelos profissionais da equipe odontológica

\begin{tabular}{lcc}
\hline $\begin{array}{l}\text { Tipos de } \\
\text { orientação e ou } \\
\text { atendimento }\end{array}$ & Freq.absol. & Freq. relat. \\
\hline \begin{tabular}{l} 
Instrução de higiene \\
\hline Escovação supervisionada
\end{tabular} & 86 & $87,8 \%$ \\
\hline $\begin{array}{l}\text { Palestras educativo- } \\
\text { preventivas à comunidade }\end{array}$ & 53 & $72,4 \%$ \\
\hline $\begin{array}{l}\text { Orientação individual } \\
\text { Reforço educativo }\end{array}$ & 82 & $54,1 \%$ \\
\hline $\begin{array}{l}\text { Distribuição de material } \\
\text { educativo }\end{array}$ & 80 & $83,7 \%$ \\
\hline Bochechos de flúor & 63 & $81,6 \%$ \\
\hline Aplicação tópica de flúor & 63 & $64,3 \%$ \\
\hline Aplicação de selante & 56 & $49,0 \%$ \\
\hline Outros & 16 & $64,3 \%$ \\
\hline
\end{tabular}

Fonte: Londrina, 1996

TABELA 7 - Distribuição das respostas dos pais referentes ao tipo de orientação e ou atendimento educativo-preventivo de cárie dentária que receberam

\begin{tabular}{lll}
\hline $\begin{array}{l}\text { Tipo de } \\
\text { orientação e ou } \\
\text { atendimento }\end{array}$ & Freq. absol. & Freq. relat. \\
$\begin{array}{l}\text { Como escovar/ } \\
\text { limpar os dentes }\end{array}$ & 377 & $91,1 \%$ \\
\hline $\begin{array}{l}\text { Passar fio dental } \\
\text { Aplicação de flüor } \\
\text { em casa }\end{array}$ & 254 & $61,4 \%$ \\
\hline $\begin{array}{l}\text { Aplicação de flúor } \\
\text { no posto }\end{array}$ & 206 & $49,8 \%$ \\
\hline $\begin{array}{l}\text { Bochechos de flúor } \\
\text { Aplicação de selante }\end{array}$ & 192 & $45,4 \%$ \\
\hline $\begin{array}{l}\text { Orientação quanto a } \\
\text { dieta alimentar }\end{array}$ & 122 & $14,0 \%$ \\
\hline Como a cárie aparece & 168 & $29.5 \%$ \\
\hline O que acontece se a & & $44,9 \%$ \\
\hline cárie não for tratada & 192 & $40,6 \%$ \\
\hline Outros & 17 & $46,4 \%$ \\
\hline
\end{tabular}


respectivamente, evidenciando que esta é uma orientação já fixada e, provavelmente, incorporada como hábito pela população. Aplicações de flúor em casa, bochechos de flúor e aplicação de selante citados na proporção $49,8 \%$, $14,0 \%$ e 29,5\% respectivamente, pelos usuários, são procedimentos aplicados em crianças dependendo da sua idade e do seu desenvolvimento psicomotor. A orientação individual $(83,7 \%)$ e o reforço educativo $(81,6 \%)$ realizados com bastante freqüência, são fundamentais no processo de cducação, visto que, o contato pessoal freqüente entre os membros da equipe de saúde e o indíviduo e sua comunidade é a maneira mais eficaz de se ter sucesso em educação para a saúde bucal(14).

TABELA 8 - Distribuição das respostas referentes a assuntos que são abordados pelos profissionais da equipe odontológica das UBSs nas orientações e ou atendimentos educativo-preventivos de cárie dentária

\begin{tabular}{llc}
\hline $\begin{array}{c}\text { Assuntos } \\
\text { abordados nas } \\
\text { orientações }\end{array}$ & Freq.absol. & Freq. relat. \\
$\begin{array}{l}\text { Etiologia da cárie } \\
\text { dentária }\end{array}$ & 52 & $53,1 \%$ \\
\hline $\begin{array}{l}\text { Como limpar/ } \\
\text { escovar os dentes }\end{array}$ & 96 & $97,6 \%$ \\
\hline $\begin{array}{l}\text { Conseqüências da } \\
\text { cárie dentárià }\end{array}$ & 87 & $88,6 \%$ \\
\hline \begin{tabular}{l} 
Dieta alimentar \\
\hline Outros
\end{tabular} & 85 & $86,7 \%$ \\
\hline & 10 & $10,2 \%$ \\
\hline
\end{tabular}

Na tabela 8 , chama atenção a baixa freqüência de pais que receberam orientações quanto à dieta alimentar $(44,9 \%)$, etiologia da cárie dentária $(40,6 \%)$ c suas consequências $(46,4 \%)$, confrontando com as respostas dos profissionais (tabela 8 ), que afirmam abordar as conseqüências da cárie numa proporção de $88,6 \%$ e quanto a dicta alimentar, $86,7 \%$, discrepância esta que sugere haver dificuldades na forma de comunicação/entendimento entre o profissional e o usuário. Quanto ao assunto etiologia da cáric dentária, este parece ser um ponto falho na rotina educativopreventiva das UBSs, pois apenas $53,1 \%$ dos profissionais esclarece este assunto, um passo fundamental no processo de conscientização. Esta conscientização só acontece quando as pessoas tomam posse da realidade, conhecendo-a. E conhecendo-a, descubram que é modificável e que podem fazê-lo(iI).

Visando avaliar se houve um real processo de ensinoaprendizagem entre profissionais e pais, foi levantada a questão se sabiam como prevenir as cáries, onde 95,3\% respondem que sim, mas quando questionados se acham possível ter dentes sem cárie a vida toda, apenas $52,4 \%$ respondem afirmativamente, conforme mostra a figura 2 . Estes dados contraditórios levantam três hipóteses: as pessoas sabem como evitar a cárie, mas acham os procedimentos difíceis, a cárie dentária é um assunto que não está totalmente claro para a pessoa ou, realmente sabem como evitar a cárie, mas consideram-na uma fatalidade.

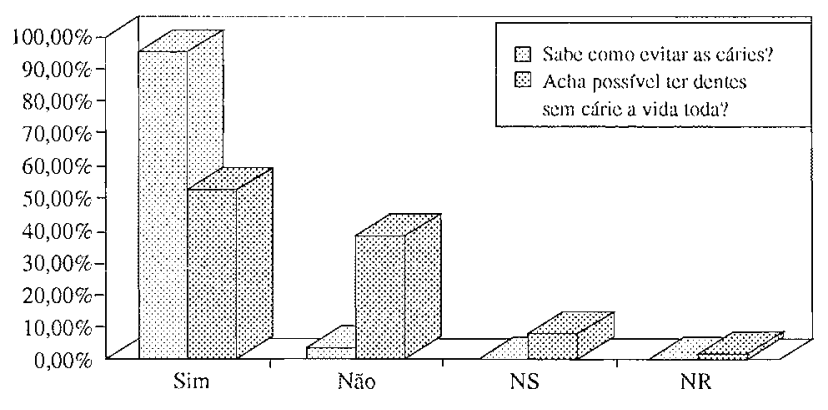

FIGURA 2 - Distribuição percentual das respostas referentes às questões: "Sabe como evitar as cáries?" e "Acha possivel ter dentes sem cáries a vida toda?"

\subsection{GRUPOS DE PRESSÃO E MUDANÇAS DE COMPORTAMENTO}

Segundo LOCKER (1989), a obtenção da cooperação de pequenos grupos de pessoas que se conhecem, como parentes, membros da família, amigos, crianças, 
proporciona um reforço positivo no cotidiano das pessoas, scrvindo como um grupo de pressão no que se refere a mudanças de comportamento.

TABELA 9 - Distribuição das respostas dos usuários referentes a onde receberam as orientaç $\nsim$ es ou

\begin{tabular}{|c|c|c|}
\hline $\begin{array}{c}\text { Onde recebeu as } \\
\text { orientaçóes e ou } \\
\text { atendimento } \\
\text { educativo- } \\
\text { preventivo }\end{array}$ & Freq. absol. & Freq. relat. \\
\hline UBS & 394 & $95,2 \%$ \\
\hline Colegas & 10 & $2,4 \%$ \\
\hline Vizinhos & 03 & $0,7 \%$ \\
\hline Familiares & 11 & $2,7 \%$ \\
\hline Televisão & 34 & $8,2 \%$ \\
\hline Folhetos, cartazes e vídeo & 46 & $11,1 \%$ \\
\hline Outros & 31 & $7,5 \%$ \\
\hline Não sabe & $0 !$ & $0,2 \%$ \\
\hline
\end{tabular}

Fonte: Londrina, 1996

Na tabela 9, quando indagados a respeito do local onde receberam estas orientações, 95,2\% apontam as UBSs, seguido por folhetos, cartazes e vídeo citados por $11,1 \%$ e televisão citado por $8,2 \%$ dos pais entrevistados. O fato dos familiares, colegas e vizinhos serem tão pouco citados $(2,7 \%, 2,4 \%$ e $0,7 \%$ respectivamente), sugere que conhecimentos a respeito da prevenção de cárie dentária não lazcm parte do cotidiano dessas pessoas. Daí a importância das UBSs na disseminação de informações a respeito da cáric, colaborando para que estes conceitos e o estímulo do auto-cuidado sejam introduzidos na cultura habitual das pessoas. Os indivíduos geralmente não mudam de atitude e comportamento, se a mudança é, ou aparenta ser, contrária a esses grupos. (i0)
TABELA 10 - Distribuição das respostas dos usuários referentes a mudanças positivas na saúde bucal da criança e no hábito da família após terem recebido orientação e ou atendimento educativo-preventivo de cárie dentária

\begin{tabular}{|c|c|c|c|c|}
\hline & \multicolumn{2}{|c|}{$\begin{array}{c}\text { Mudança na } \\
\text { saúde bucal da } \\
\text { criança }\end{array}$} & \multicolumn{2}{|c|}{$\begin{array}{c}\text { Mudança no } \\
\text { hábito da família }\end{array}$} \\
\hline & Freq.ab & Freq. relat. & Freq.abs & Freq. relat \\
\hline $\operatorname{Sim}$ & 353 & $85,3 \%$ & 310 & $74,9 \%$ \\
\hline Não & 51 & $12,3 \%$ & 85 & $20,5 \%$ \\
\hline Não responde & 02 & $0,5 \%$ & 09 & $2,2 \%$ \\
\hline Năo sabe & 08 & $1,9 \%$ & 10 & $2.4 \%$ \\
\hline TOTAL & 414 & $100 \%$ & 414 & $100 \%$ \\
\hline
\end{tabular}

Na tabela 10 , nota-se um significativo número de pais $(85,3 \%)$ que relatam ter havido mudanças posilivas na saúde bucal da criança após terem recebido oricntação e ou atcndimento educativo-preventivo de cárie dentária. 0 fato de terem percebido mudanças positivas na saúdo bucal de suas crianças torna-se importante, na mediưa en que a avaliação da aceitação do uso de programas e medidas preventivas deve ser baseado em mudanças do comportamento e não apenas cm conhecimento adquirido(8), além de coopcrar no processo de educação pois, conforme os pais percebem que ier saúde bucal é algo concreto, sentem-se cada vez mais estimulados a realizá-lo.

Na tabela 10, a ocorrência de mudanças nos hábitos bucais da família após atendimento na UBS é relatado por $74,9 \%$ dos pais. Estes resultados são também verificados por NAKAMA (1994) onde $60,6 \%$ dos pais entrevistados que mantêm seus filhos na Bcbê-Clínica relatam ter havido mudanças positivas no hábito familiar. Estes dados comprovam que as crianças constituem um importante grupo de pressão na mudança de hábitos na família, seja passivamente, quando os pais são obrigados a modificarcmse para beneficiar seus filhos, seja ativamente, quando a criança cobra a mudança de comportamento dos pais. HOLM (1990), já afirmava que os pais podem mudar seus hábitos para beneficiar os filhos. 
TABELA 11 - Distribuição das respostas referentes a opinião dos profissionais da equipe odontológica a respcito da eficácia de seu trabalho educativo-preventivo

\begin{tabular}{lcc}
$\begin{array}{l}\text { Considera seu } \\
\text { trabalho eficaz }\end{array}$ & Freq. absol. & Freq. relat. \\
\hline Sim & 90 & $91,8 \%$ \\
\hline Não & 08 & $8,2 \%$ \\
\hline TOTAL & 98 & $100,0 \%$ \\
\hline & & Fonte: Londrina, 1996
\end{tabular}

Na tabela 11,quando analisa-se a opinião dos profissionais da equipe odontológica quanto a eficácia de seu trabalho educativo-preventivo, 91,8\% consideram-no elicaz. Este fato é positivo, à medida que o bom trabalho em equipe depende essencialmente da confiança das pessoas no trabalho que realiza, ou seja, não há resultados positivos quando as pessoas não acreditam no que fazem.

\section{CONCLUSÃO}

Após o cstudo dos três componentes diretamente envolvidos no processo de educação para a sáude bucal nas UBSs de Londrina, pode-se concluir que:

a) Os fatores psicossociais: caracterização sóciocconômica, percepções e crenças, conhecimentos e informação, grupos de pressão e mudança de comportamento, podem interferir de maneira significativa no processo educacional e devem, portanto, ser estudados com critério, a fím de se traçar um perfil real da população alvo para se estabelecer um programa de saúde bucal clicaz na promoção e manutenção da saúde bucal.

b) As UBSs têm produzido mudanças comportamentâis positivas em relação à saúde bucal e ao saber das pessoas, evidenciando que a mudança no vetor de cducação (criança para pais) está beneficiando não apenas crianças de 0 a 14 anos, mas, por extensão,uma parcela da população adulta. No entanto, crenças negativas sobre a cárie, etiologia da cáric dentária e suas consequîencias, bem como a dieta alimentar são questões que poderiam ser melhor trabalhadas pelas equipes.

c) A atuação da eqquipe odontológica está dentro da filosofia educativo-preventiva adotada pela Secretaria de Saúde, mostrando também a importância do trabalho dos THDs e ACDs nesse processo, sugerindo que haja maior incentivo à formação e inserção destes profissionais na equipe odontológica, pois para funções específicas de educação para a saúde, se ampliaria a cobertura à população a um baixo custo para o município.

\section{REFERÊNCIAS BIBLIOGRÁFICAS}

1.BJARNASON $S$ et al. Caries experience in Icelandic 12-year-old urbanchildren between 1984 and 1991. Community Dent Oral Epidemiol, v.21, p.194-197, 1993.

2.CHAVES, Mario M. Odontologia Social. $3^{\circ}$ ed. São Paulo: Artes Médicas, 1986.

3.FEJERSKOV, O. Strategies in the Design of Preventive Programs. Advances in Dental Research, v.9, n.2, p. 82-88, july.1995.

4.GIANINI, M. Conferência II: "Papel dos NãoDentistas". ABOPREV, [S.1.], p.6, abr/mai/jun.1995.

5.HOLM,A.K. Education and diet in the prevention of caries in the preschool child. J.Dent.,Bristol, v.18, n.6, p.308-314, dec.1990.

6.HOROWITZ, A.M. The Public's Oral Health: The Gaps Between What We Know and What We Practice. Advances in Dental Research, v.9, n.2, p. 91-95, july. 1995. 
7.LOCKER, D. Behavioral Science \& Dentistry. $1^{\circ}$. ed. Great Britain: Tavistock/Routledge, 1989.

8.MATHEWSON, R.J.; PRIMOSCH, R.E.; ROBERTSON, D. Fundamentals of dentistry for children. Chicago: Quintessence, 1982.

9.MINISTÉRIO DA SAÚDE. Levantamento Epidemiológico em Saúde Bucal: Brasil, zona urbana, 1986. Brasília: Centro de Doc. do Ministério da Saúde, 1988.

10.MORAES,N.; BIJELLA, V.T. Educação Odontológica do Paciente. Rev.da Ass. Paul. Cirurg. Dent. São Paulo, v.36, n.3, p.330-307, mai/jun. 1982.

11.NAKAMA, L. Educar prevenindo e prevenir educando - Odontologia no primeiro ano de vida. Londrina: UEL, 1994. Tese (Mestrado em Saúde Coletiva) - Curso de Odontologia- Universidade Estadual de Londrina.

12. PETERSEN, P.E.; DANILA I.; SAMOILA A. et.al. Oral health behavior, knowledge, and attitudes of children, mothers, and school teachers in Romania in 1993. Acta Odontol Scand, v.53, n.6, p.363368 , dec. 1995.

13.PINTO, V.G. Índice de Cárie no Brasil e no Mundo. Revista Gaúcha de Odontologia. Porto Alegre, v.44, n.l, p.8-14, jan/fev. 1996.

14. PINTO, V.G. Saúde Bucal. $3^{\circ}$ cd. São Paulo: Santos, 1992. cap.9: Educação em Saúde, p.235.

15.PREFEITURA DO MUNICÍPIO DE LONDRINASERVIÇO MUNICIPAL DE SAÚDE. Programa de Orientação e Educação em Saúde Bucal. Londrina, [199-]. 